\title{
COVERAGE OF A SQUARE LATTICE BY AN INCLINED RECTANGLE
}

\author{
DENNIS ROSEN, ${ }^{*}$ Birkbeck College, London
}

\begin{abstract}
By use of a method of dissection, a formula is derived for the variance in the number of lattice points covered by a rectangle of arbitrary size, lying on a square lattice of unit spacing and inclined to the lattice at an angle of which the tangent is a rational fraction.
\end{abstract}

GEOMETRIC PROBABILITY; COVERAGE

\section{Introduction}

Kendall and Moran (1963) quote a formula for the variance in the coverage of a square lattice by a rectangle of arbitrary size, randomly positioned, but with its sides parallel to the two directions of the lattice. However, they note the absence of a corresponding formula for the case where the orientation of the rectangle is also random. The result presented here gives the variance when the rectangle is randomly positioned at a fixed angle to the lattice, with the tangent of the angle a rational fraction; it is obtained by dissecting the rectangle into appropriate regions for which the variances and covariances can be calculated. Although falling far short of the goal of dealing with a randomly oriented rectangle, the result is of use in tasks of automated image analysis involving mensuration of rectangular areas.

\section{Areas of constant counting measure}

With respect to a square lattice with points specified by integer pairs $\left(x_{i}, y_{i}\right)$, the counting measure of a set is the number of lattice points covered. The lines passing through precisely the lattice points $\left(a_{0}+n a, b_{0}+n b\right)$ with $a, b$ coprime and $n=0, \pm 1, \pm 2, \cdots$, will be called $\theta$-lines, with $\theta=\tan ^{-1}(a / b)$. The lines make an angle $\theta$ with the $x$-axis. The distance between consecutive points on a $\theta$-line is $D=\left(a^{2}+b^{2}\right)^{\frac{1}{2}}$ and the perpendicular distance between adjacent $\theta$-lines is $U=(\cos \theta) / b=\left(a^{2}+b^{2}\right)^{-\frac{1}{2}}=1 / D$. A parallelogram will be taken to include its left-hand edge with both ends and its lower edge with its left-hand end, but exclude the rest of the boundary.

Lemma. Every parallelogram with a pair of sides of length $D$, inclined to the $x$-axis at an angle $\theta$, and separated by a distance $U$, contains exactly one lattice point.

Proof. Each such parallelogram is intersected by exactly one $\theta$-line, on which it cuts off an intercept of length $D$. Hence it contains exactly one lattice point.

Thus a parallelogram with the charcteristics just stated is a set of constant measure, namely of unit measure. When such a parallelogram is a rectangle it will be referred to as a standard unit rectangle. The union of disjoint sets of constant measure has constant measure.

\section{The measure of an inclined rectangle}

For a rectangle of sides $L_{1}$ and $L_{2}$, inclined to the $x$-axis of the lattice at angle $\theta=\tan ^{-1}(a / b)$, the sides may be written as $L_{1}=r_{1} D+s_{1} U+V_{1}$, and $L_{2}=r_{2} D+s_{2} U+V_{2}$ where $D$ and $U$ are defined as above, $r_{i}, s_{i}$ are integers and $V_{i}<U \leqq 1$ since $D \geqq 1$. Thus the

Received 10 October 1988; revision received 4 May 1989.

* Postal address: Department of Crystallography, Birkbeck College, Malet Street, London, WC1E 7HX, UK. 


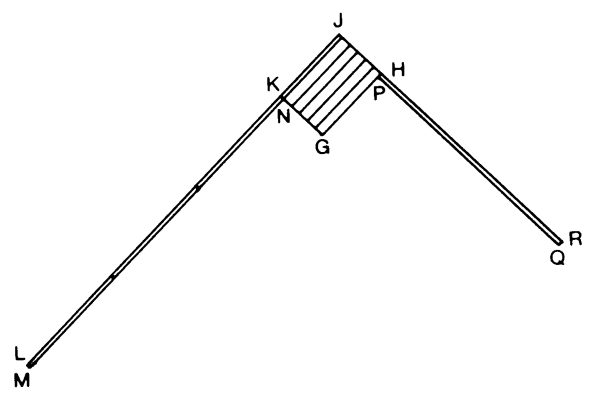

Figure 1. $L J=L_{1}, L K=r_{1} D, K J=M_{1}=S_{1} U+V_{1}: R J=L_{2}, R H=r_{2} D, H J=M_{2}=s_{2} U+V_{2}$.

rectangle is decomposed into $r_{1} r_{2}$ squares of side $D$ and of measure $D^{2}, s_{1} r_{2}+s_{2} r_{1}$ standard unit rectangles and a remaining area, illustrated in Figure 1, which can be considered in terms of its constituent regions. Region I, $K L M N$ in Figure 1, consists of $r_{1}$ strips of length $D$ and width $V_{2}$ lying end to end and parallel to side $L_{1}$ of the rectangle. Region II, $P Q R H$ in Figure 1 , consists of $r_{2}$ strips of length $D$ and width $V_{1}$, lying end to end and parallel to side $L_{2}$ of the rectangle. The final part of the remaining area, $G H J K$ in Figure 1, is a small rectangle of sides $M_{1}=s_{1} U+V_{1}$ and $M_{2}=s_{2} U+V_{2}$ and can be decomposed either as $s_{2}$ strips of length $M_{1}$ and width $U$ together with one of length $M_{1}$ and width $V_{2}$ or else as $s_{1}$ strips of length $M_{2}$ and width $U$ together with one of length $M_{2}$ and width $V_{1}$. It is immaterial which decomposition is used, although for numerical calculation it is convenient to take that which includes the smaller number of strips. Suppose that $s_{2}<s_{1}$ so that the first decomposition is taken, as illustrated in Figure 1. Then it is convenient to consider Region III as consisting of $s_{2}$ strips of length $M_{1}$ and width $U$ and Region IV as the single strip of length $M_{1}$ and width $V_{2}$. The mean measures and the variances and covariances of and between the four regions can now be calculated by integrating over a standard unit rectangle .

By applying elementary methods it immediately follows that the mean measure $\mu$ of the whole rectangle equals its area, that the variances of Regions I, II and IV are $r_{1}^{2} D V_{2}(1-$ $\left.D V_{2}\right), r_{2}^{2} D V_{1}\left(1-D V_{1}\right)$ and $M_{1} V_{2}\left(1-M_{1} V_{2}\right)$ respectively, the covariance, cov (I, II), between Regions I and II is zero, $\operatorname{cov}(\mathrm{I}, \mathrm{IV})=r_{1} M_{1} V_{2}\left(1-D V_{2}\right)$ and $\operatorname{cov}(\mathrm{II}, \mathrm{IV})=r_{2} V_{1} V_{2}\left(1-D V_{1}\right)(1-$ $\left.M_{1} V_{2}+V_{1} V_{2}\right)$.

The variance of Region III involves cov $(i, j)$, the covariance between the $i$ th and $j$ th strips. The latter may be calculated by moving the rectangle so that $G$, the bottom left-hand corner of the first strip (Figure 1) is a lattice point and then rotating axes about $G$ so that the new axes $(X, Y)$ are inclined at angle $\theta$ to the old $(x, y)$. The $s_{2}$ strips of Region III are fractions, $M_{1} / D$, of standard unit rectangles, in the $i$ th one of which the lattice point is at $\left(X_{i}, Y_{i}\right)$ where $X_{i}=y_{i} \sin \theta+x_{i} \cos \theta, Y_{i}=y_{i} \cos \theta-x_{i} \sin \theta=i / D$ and $x_{i}, y_{i}$ are appropriate solutions of the Diophantine equation

$$
y_{i} b-x_{i} a=i .
$$

With $E_{i j}=\left|X_{j}-X_{i}\right|, F_{i j}=D-E_{i j}$, and $G_{i j}$ equal to the lesser of $E_{i j}$ and $F_{i j}$, it then follows that

$$
\begin{array}{ll}
\text { for } M_{1} \leqq E_{i j}, & \operatorname{cov}(i, j)=-M_{1}^{2} / D^{2} ; \\
\text { for } E_{i j} \leqq M_{1} \leqq F_{i j}, & \operatorname{cov}(i, j)=\left(D M_{1}-D G_{i j}-M_{1}^{2}\right) / D^{2} ; \\
\text { for } M_{1} \geqq F_{i j}, & \operatorname{cov}(i, j)=-\left(D-M_{1}\right)^{2} / D^{2} .
\end{array}
$$

The covariances between the strips of Region III and that of Region IV can be written as $\operatorname{cov}\left(i, s_{2}\right)$ and have values equal to the corresponding $\operatorname{cov}(i, j)$ values multiplied by $V_{2} D$.

The covariance between Region I and each strip of Region III is zero. The covariance between Region II and each strip of Region III is $r_{2} V_{1}\left(1-D V_{1}\right)\left(D-M_{1}-V_{1}\right) / D^{2}$. 
Since $\operatorname{var}(m)$, the variance on the measure, $m$, of the rectangle, is given by $\Sigma$ var $(l)+$ $2 \Sigma \operatorname{cov}(l, m)$ where $l, m=$ I, II, III, IV, then on collecting terms and rearranging,

$$
\begin{aligned}
\operatorname{var}(m)= & V_{2}\left(1-D V_{2}\right) L_{1}^{2} / D+V_{1}\left(1-D V_{1}\right) L_{2}^{2} / D \\
& +M_{2}\left\{M_{1}\left(1-M_{1} U\right)-U V_{1}\left(1-D V_{1}\right)\left(2 r_{2} M_{1}-2 r_{2} V_{1}+M_{2}\right)\right\} \\
& +2 \Sigma \operatorname{cov}(i, j)+2 \Sigma \operatorname{cov}\left(i, s_{2}\right),
\end{aligned}
$$

where $\operatorname{cov}(i, j)$ and $\operatorname{cov}\left(i, s_{2}\right)$ are given by (2) and the modification of (2) as noted. The case where $s_{2}=0$ is included in (3) if the last two terms are then taken as zero.

\section{Comments on the results}

If $M_{1}$, the remainder of $L_{1} / D$, is less than or equal to $\sin \theta$, then

$$
2 \Sigma \operatorname{cov}(i, j)+2 \Sigma \operatorname{cov}\left(i, s_{2}\right)=-\left(s_{2}^{2}-s_{2}+2 D V_{2}\right) M_{1}^{2} / D_{2}
$$

and if $M_{1} \geqq D-\sin \theta$ then

$$
2 \Sigma \operatorname{cov}(i, j)+2 \Sigma \operatorname{cov}\left(i, s_{2}\right)=-\left(s_{2}^{2}-s_{2}+2 D V_{2}\right)\left(D-M_{1}\right)^{2} / D_{2} \text {. }
$$

Except for these cases, (3) must be evaluated after collecting solutions to (1) with $i=0,1,2, \cdots, s_{2}$, although with some combinations of $L_{1}$ and $\theta,(4)$ and (5) will also be true with less restricted ranges of $M_{1}$, thereby simplifying the formula for $\operatorname{var}(m)$. The variables which may be set, that is, the size of the rectangle through $L_{1}, L_{2}$ and its inclination to the lattice through $\theta=\tan ^{-1}(a / b)$ make $\operatorname{var}(m)$ vary in a highly discontinuous way.

When the rectangle is aligned with the lattice so that $\theta$ and $a$ are zero, $b=D=u=1$, $r_{2}=s_{2}=0$ and $M_{1} M_{2}$ reduce to $V_{1}, V_{2}$, (3) reduces to the formula of Kendall and Moran (1963), namely

$$
\operatorname{var}(m)=V_{2}\left(1-V_{2}\right) L_{1}^{2}+V_{1}\left(1-V_{1}\right) L_{2}^{2}+V_{1} V_{2}\left(1-V_{1}\right)\left(1-V_{2}\right) .
$$

If the formula derived here is used in image analyses of digitized images then care must be taken, as previously noted (Rosen (1980)), to distinguish between lattice and grid. In the processing of digitized images, it is usual to work with pixels (picture elements) and if these are taken to lie in a lattice then the edges of the area formed by a group of pixels lie on a grid which transects the lattice.

\section{References}

Kendall, M. and Moran, P. A. P. (1963) Geometrical Probability. Griffin, London.

Rosen, D. (1980) On the areas and boundaries of quantized objects. Comp. Graphics Image Proc. 13, 94-98. 